\title{
Trophic magnification of short-chain per- and polyfluoroalkyl substances in a terrestrial food chain from the Tibetan Plateau
}

Kai Huang, ${ }^{\# 1,2}$ Yili Li ${ }^{\#}, 2$ Duo Bu, ${ }^{3}$ Jie Fu, ${ }^{1,2}$ Meilin Wang, ${ }^{2}$ Wei Zhou, ${ }^{1,2}$ Luyao Gu, ${ }^{1,2}$ Yilin Fu, ${ }^{2}$ Zhiyuan Cong, ${ }^{4}$ Boyuan Hu, ${ }^{1}$ Jianjie Fu*, ${ }^{1,2,3,5}$ Aiqian Zhang, ${ }^{1,2,5}$ and Guibin Jiang $1,2,3$

${ }^{1}$ School of Environment, Hangzhou Institute for Advanced Study, UCAS, Hangzhou, 310024, China

${ }^{2}$ State Key Laboratory of Environmental Chemistry and Ecotoxicology, Research Center for Eco-Environmental Sciences, Chinese Academy of Sciences, Beijing, 100085, China

${ }^{3}$ Department of Chemistry \& Environmental Science, Tibet University, Lhasa, 850000, China

${ }^{4}$ Key Laboratory of Tibetan Environment Changes and Land Surface Processes, Institute of Tibetan Plateau Research, Chinese Academy of Sciences, Beijing, 100101, China

${ }^{5}$ Institute of Environment and Health, Jianghan University, Wuhan, 430056, China

Corresponding author:

Dr. Jianjie Fu

Tel: +86-10-6284-9357

Fax: +86-10-6284-9339

E-mail:jjfu@rcees.ac.cn

Research Center for Eco-Environmental Sciences,

Chinese Academy of Sciences, Beijing 100085, China

\section{Text: 7}

Table: 8

Figure: 4

Page: 21 


\section{Table of Contents}

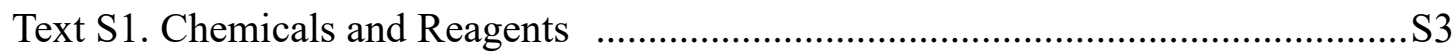

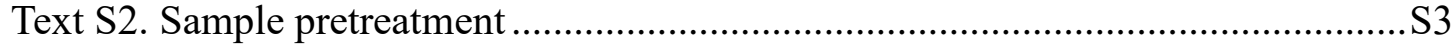

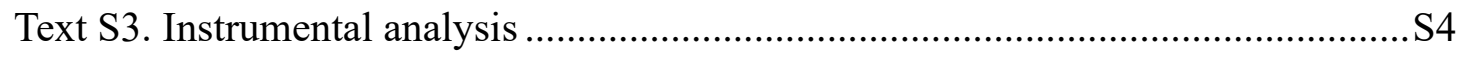

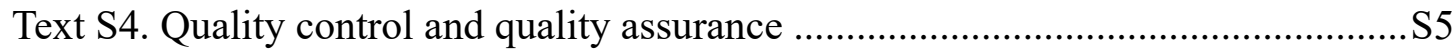

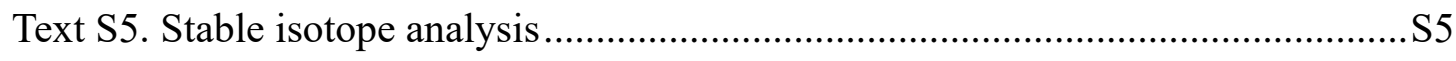

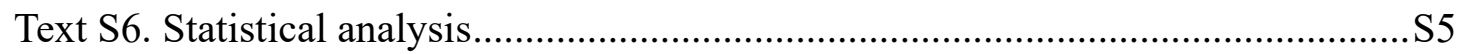

Text S7. Confirmation of PFAS quantification by Orbitrap MS..................................S6

Table S1. List of native and mass-labelled PFASs.................................................... 8

Table S2. Optimized MS parameters for analysis of PFASs...................................... 9

Table S3. Method detection limits (MDLs) of PFASs in different sample types .......S10

Table S4. Detection frequencies (\%) of PFASs in different sample types.................S11

Table S5. Mean concentrations and concentration ranges of PFASs in different sample

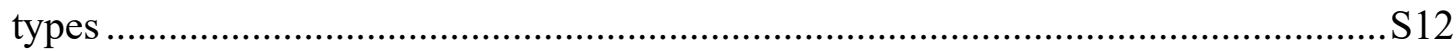

Table S6. Comparison of concentration results ( $\mathrm{ng} / \mathrm{g} \mathrm{dw}$ ) obtained from triple quadruple

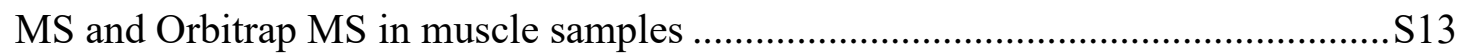

Table S7. Stable isotopes and trophic levels of the plant-pika-eagle food chain.......S14

Table S8. TMFs of PFASs in different types of food webs in different regions ........S15

Figure S1. Confirmation analysis of PFOS in muscle samples by Orbitrap MS.......S16

Figure S2. Confirmation analysis of PFBA in muscle samples by Orbitrap MS.......S17

Figure S3. Correlations between PFASs concentrations (ng/g dw) in eagle muscles and

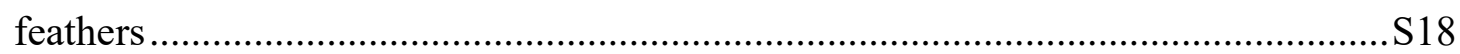

Figure S4. Relationships between TMFs and physicochemical properties ...............S19

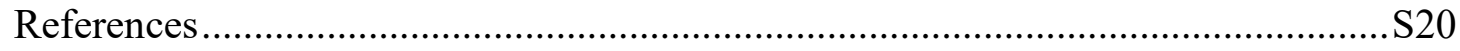




\section{Text S1. Chemicals and Reagents}

Native and mass-labelled PFASs standard solutions were purchased from Wellington Laboratories (Guelph, ON, Canada). Native PFASs were composed of 10 PFCAs $\left(\mathrm{C}_{4-}\right.$ $\left.\mathrm{C}_{13}\right)$ and 3 PFSAs $\left(\mathrm{C}_{4}, \mathrm{C}_{6}\right.$ and $\left.\mathrm{C}_{8}\right)$; mass labelled standards included 9 PFCAs $\left(\mathrm{C}_{4}-\mathrm{C}_{12}\right)$ and 3 PFSAs $\left(\mathrm{C}_{4}, \mathrm{C}_{6}\right.$ and $\left.\mathrm{C}_{8}\right)$. Full names and abbreviations of native and mass-labelled PFASs are listed in Table S1. HPLC grade methanol (MeOH) and acetonitrile (ACN) were purchased from Fisher Scientific (Waltham, MA, USA). Ultrapure water was obtained from a Milli-Q Integral 5 system. Ammonium acetate $\left(\mathrm{NH}_{4} \mathrm{Ac}\right)$ and ammonium hydroxide $\left(\mathrm{NH}_{4} \mathrm{OH}, 28 \%\right)$ were purchased from Fisher Scientific (Waltham, MA, USA). HPLC grade formic acid (FA) was purchased from Dikma Technologies (Bejing, China). Oasis WAX cartridges $(6 \mathrm{~mL}, 150 \mathrm{mg})$ were purchased from Waters (Milford, MA, USA). Supelclean ENVI-Carb cartridges (6 mL, $500 \mathrm{mg}$ ) were purchased from Supelco (St. Louis, MO, USA).

\section{Text S2. Sample pretreatment}

The pretreatment methods of muscle samples were optimized to minimize extraction losses and matrix effects. ACN containing $0.1 \%$ FA was chosen as the extraction solvent Briefly, an aliquot of $0.2 \mathrm{~g}$ of muscle sample was weighed into a $15 \mathrm{~mL}$ polypropylene tube and $5 \mathrm{ng}$ of internal standards was mixed. $10 \mathrm{~mL}$ of extraction solvent was added and the mixture was rotated for $16 \mathrm{~h}$. Samples were centrifuged at $3500 \mathrm{rpm}$ for 10 minutes and the resulting supernatant was decanted into a new polypropylene tube. Another $3 \mathrm{~mL}$ of extraction solvent was used to extract the residues for $30 \mathrm{~min}$ and the procedure was repeated twice. The extract was combined, concentrated to $1 \mathrm{~mL}$ and diluted with $36 \mathrm{~mL}$ of water for clean-up. Oasis WAX cartridges $(6 \mathrm{~mL}, 150 \mathrm{mg})$ were preconditioned with $4 \mathrm{~mL} \mathrm{MeOH}$ containing $0.1 \% \mathrm{NH}_{4} \mathrm{OH}, \mathrm{MeOH}$ and water respectively prior to sample introduction. After sample loading, the cartridges were cleaned with $4 \mathrm{~mL} \mathrm{NH}_{4} \mathrm{Ac}$ solution $(25 \mathrm{mM})$, dried by centrifugation and eluted with 4 $\mathrm{mL} \mathrm{MeOH}$ and $4 \mathrm{~mL} \mathrm{MeOH}$ containing $0.1 \% \mathrm{NH}_{4} \mathrm{OH}$. The extracts were dried to $1 \mathrm{~mL}$ under $\mathrm{N}_{2}$ and centrifuged at $12,000 \mathrm{rpm}$ for ten minutes to remove residues. Feather samples were treated according to the previously reported method. ${ }^{1}$ Soil and plant 
samples were also extracted by ACN containing 0.1\% FA as described above, but cleaned with ENVI-Carb cartridges to remove pigments. In brief, the combined extraction solvent was concentrated to approximately $1 \mathrm{~mL}$ for clean-up. After rinsed with $4 \mathrm{~mL} \mathrm{MeOH}$, the extracts were loaded to ENVI-Carb cartridges (6 mL, $500 \mathrm{mg}$ ) and eluted with $4 \mathrm{~mL} \mathrm{MeOH}$ and $4 \mathrm{~mL} \mathrm{MeOH}$ containing $0.1 \% \mathrm{NH}_{4} \mathrm{OH}$. Fractions were concentrated to approximately $1 \mathrm{~mL}$ and centrifugated prior to analysis.

\section{Text S3. Instrumental analysis}

PFASs were analyzed by an Ultimate 3000 high performance liquid chromatography (HPLC) system coupled with a TSQ Quantiva Triple Quad mass spectrometer (Thermo Fisher Scientific, USA). Analytes were separated on an Acclaim 120 C18 column (5 $\mu \mathrm{m}, 4.6 \mathrm{~mm}$ i.d., $150 \mathrm{~mm}$ length; Dionex, USA). The mobile phase was composed of ACN (A) and $\mathrm{H}_{2} \mathrm{O}$ with $10 \mathrm{mM} \mathrm{NH}_{4} \mathrm{Ac}(\mathrm{B})$ with a flow rate of $1 \mathrm{~mL} / \mathrm{min}$. A gradient elution program was set as follows: $20 \% \mathrm{~B}$ was increased to $95 \%$ B over 4 minutes and held for 3 minutes; then returned to initial condition $(20 \% \mathrm{~B})$ over 1 minute and held for another 2 minutes. The column temperature was set to be $40^{\circ} \mathrm{C}$ and the injection volume was $10 \mu \mathrm{L}$. Data were acquired using selective reaction monitoring (SRM) under negative ionization mode. Optimized MS parameters are presented in Table S2. Interferences caused by endogenous compounds (like bile acids or fat acids) in quantification of PFOS, PFHxS and PFBA by triple quadruple MS in biological samples were reported. ${ }^{2,3}$ These endogenous compounds might also present in muscle samples and thereby cause biased quantification results of PFASs. To identify if there were interferents coeluting with these PFASs, muscle samples were tested using Q Exactive Orbitrap Mass Spectrometer (Thermo Fisher Scientific, USA). Confirmation analysis was conducted on Orbitrap MS by using pika and eagle muscle samples. The analytical column and mobile phase condition were the same as those used in triple quadruple MS analysis. The Orbitrap was operated using full scan (mass range: 200$1200 \mathrm{~m} / \mathrm{z}$, resolution $=140,000)$ in negative ionization mode. Specially, for confirmation of PFBA, data-dependent $\mathrm{MS}^{2}$ scan was triggered by the detection of accurate mass of PFBA $(\mathrm{m} / \mathrm{z}=212.9781)$ with a mass resolution of 17,500 and collision 
energy of $35 \mathrm{eV}$. Capillary voltage was $3.0 \mathrm{kV}$ and capillary and vaporizer temperatures were $320^{\circ} \mathrm{C}$ and $350^{\circ} \mathrm{C}$ respectively. Quantification was also performed using native and internal standards.

\section{Text S4. Quality control and quality assurance}

Polytetrafluoroethylene (PTFE) materials were avoided during all the courses of sample collection, preparation, and instrumental analysis. Procedural blanks were prepared with every sample set and solvent blanks using ACN were injected between samples to monitor carryover and background contamination. There were no apparent contaminations of PFASs in both procedural and solvent blanks. A calibration standard was injected after every 10 injections for checking instrumental drift. Quantification was conducted based on a 9-point calibration curve using isotope dilution method. Recoveries of PFASs in all sample types were in the range from $72 \%$ to $121 \%$. Method detection limits (MDLs) of PFASs for all sample types are listed in Table S3.

\section{Text S5. Stable isotope analysis}

Stable nitrogen $\left(\delta^{15} \mathrm{~N}\right)$ and carbon $\left(\delta^{13} \mathrm{C}\right)$ isotopes analysis was performed to estimate the trophic levels of biota samples. Samples (plants, pika and eagle muscles) were weighted (1-2 mg) and analyzed on a DELTA V ADVANTAGE isotope ratio mass spectrometer interfaced with a Flash EA1112 HT elemental analyzer (Thermo Fisher Scientific, USA). Atmospheric nitrogen and PDB (Pee Dee Belemnite) were used as isotope ratio standards for $\mathrm{N}$ and $\mathrm{C}$, respectively. Stable isotope abundances were expressed in parts per thousand (\%) according to the following equation:

$$
\begin{aligned}
\delta^{15} N & =\left(\left({ }^{15} N /{ }^{14} N\right)_{\text {sample }} /\left({ }^{15} N /{ }^{14} N\right)_{\text {standard }}-1\right) \times 1000(\% 0) \\
\delta^{13} C & =\left(\left({ }^{13} C /{ }^{12} C\right)_{\text {sample }} /\left({ }^{13} C /{ }^{12} C\right)_{\text {standard }}-1\right) \times 1000(\% 0)
\end{aligned}
$$

\section{Text S6. Statistical analysis}

All statistical analyses were performed on the R program (version 4.0.0, the $\mathrm{R}$ project for statistical computing). Concentrations below MDL were assigned to MDL/2 to calculate the mean concentrations. Student's t-tests were used to compare the 
differences between the concentrations among different species. Correlation analysis of PFASs in different samples were conducted by Pearson's correlation coefficients. Twotailed tests with a significance level of 0.05 were used for all data analyses.

The trophic levels (TLs) can be derived from the stable isotope ratio of $\delta^{15} \mathrm{~N}$ with the following relationship: ${ }^{4}$

$$
T L=1+\left(\delta^{15} N_{\text {consumer }}-\delta^{15} N_{\text {plant }}\right) / \Delta^{15} N
$$

where $\Delta^{15} \mathrm{~N}$ is the trophic enrichment factor constant, assigned as $3.4 \%$ in this study for biomagnification assessments. The trophic level of plant is assumed to be 1 .

TL-normalized biomagnification factors (BMFs) were calculated based on the following equation: ${ }^{5}$

$$
B M F=\frac{\left(C_{\text {predator }} / C_{\text {prey }}\right)}{\left(T L_{\text {predator }} / T L_{\text {prey }}\right)}
$$

where $\mathrm{C}_{\text {predator }}$ and $\mathrm{C}_{\text {prey }}$ are the concentrations of PFASs (ng/g dw) in the predator and the prey. $\mathrm{TL}_{\text {predator }}$ and $\mathrm{TL}_{\text {prey }}$ are the TLs for the predator and prey.

The standard errors (SE) of BMFs were calculated from the means and standard deviations (SD) of TL-normalized PFASs concentrations in the predator and the prey, using the following equation: ${ }^{6}$

$$
S E=\frac{B M F \times \sqrt{\left(S D_{\text {predator }} / \text { mean }_{\text {predator }}\right)^{2}+\left(S D_{\text {prey }} / \text { mean }_{\text {prey }}\right)^{2}}}{\sqrt{N_{\text {predator }}+N_{\text {prey }}}}
$$

Trophic magnification factors (TMFs) were obtained by fitting a linear relationship between log-transformed concentrations of PFASs (C, ng/g dw) and TLs, ${ }^{5}$ using the following equation:

$$
\begin{gathered}
\ln C=a+b \times T L \\
T M F=e^{b}
\end{gathered}
$$

where $a$ is the intercept and $b$ is the slope of the linear equation. The TMF can then be calculated based on equation (S7).

\section{Text S7. Confirmation of PFAS quantification by HRMS}

Confirmation analysis for individual PFASs was conducted qualitatively and quantitatively by Orbitrap MS. For quantification of PFOS on triple quadruple MS, 
499>99 transition was recommended because bile acids also generated 499>80 transition, which might cause interferences for PFOS quantification. ${ }^{2}$ Two interfering peaks in muscle samples were observed when using 499>80 transition, while no interfering peaks were observed for 499>99 transition (Figure S1A). Nevertheless, the interfering peaks can be fully separated from PFOS in the 499>80 transition. Quantification data using both the two transitions were compared and the differences were found $<7 \%$ (Table S6). So, we still chose 499>80 transition for quantification of PFOS on triple quadruple MS in this study because of its higher sensitivity. Results from Orbitrap MS showed that the interfering compound taurodeoxycholate did not coelute with PFOS (Figure S1B). The intensity of the interfering ion $(499.2933 \mathrm{~m} / \mathrm{z})$ was negligible compared with that of PFOS $(<1 \%)$ (Figure S1C).

A more recent study reported interferences of PFBA when using the only 213>169 transition. ${ }^{3}$ We also observed several interfering peaks whose retention times were longer than PFBA in the 213>169 transition (Figure S2A). To identify the interferent ions, we extracted ion chromatograms of Orbitrap MS data with a mass range of $213 \pm$ $0.5 \mathrm{~m} / \mathrm{z}$ to simulate the scan data created by triple quadruple MS (Figure S2B). The chromatographic peak at 5.22 min was PFBA, which had a monoisotopic mass of $212.9791 \mathrm{~m} / \mathrm{z}$ and a fragment ion of 168.9887 (Figure S2C). No other obvious interferent ions were observed in either full mass or $\mathrm{MS}^{2}$ scans at retention time of 5.22 min. The interferent peak at retention time of $5.40 \mathrm{~min}$, whose intensity was relatively lower than that of PFBA, was identified to have a monoisotopic mass of $213.1129 \mathrm{~m} / \mathrm{z}$ and a fragment ion of $169.1229 \mathrm{~m} / \mathrm{z}$ (Figure S2D), sharing the same transition with PFBA on triple quadruple MS. Although the interferent could not be distinguished by 213>169 transition on triple quadruple MS, it would be separated chromatographically from PFBA under the current mobile phase condition (Figure S2A).

In addition to qualitatively checking the chromatographic peaks of PFASs on Orbitrap MS, we further quantified all the 13 PFASs using accurate masses of these analytes. The quantification results showed no apparent biases $(<9.8 \%)$ between triple quadruple MS and Orbitrap MS (Table S6). 
Table S1. List of native and mass-labelled PFASs.

\begin{tabular}{|c|c|c|}
\hline & Full name & Abbreviation \\
\hline \multirow[t]{13}{*}{ Native PFASs } & Perfluorobutanoic acid & PFBA \\
\hline & Perfluoropentanoic acid & PFPeA \\
\hline & Perfluorohexanoic acid & PFHxA \\
\hline & Perfluoroheptanoic acid & PFHpA \\
\hline & Perfluorooctanoic acid & PFOA \\
\hline & Perfluorononanoic acid & PFNA \\
\hline & Perfluorodecanoic acid & PFDA \\
\hline & Perfluoroundecanoic acid & PFUnDA \\
\hline & Perfluorododecanoic acid & PFDoDA \\
\hline & Perfluorotridecanoic acid & PFTriDA \\
\hline & Perfluorobutanesulfonic acid & PFBS \\
\hline & Perfluorohexanesulfonic acid & PFHxS \\
\hline & Perfluorooctanesulfonic acid & PFOS \\
\hline Mass-labelled & {$\left[{ }^{13} \mathrm{C}_{4}\right]$-Perfluorobutanoic acid } & MPFBA \\
\hline \multirow[t]{11}{*}{ PFASs } & {$\left[{ }^{13} \mathrm{C}_{3}\right]$-Perfluoropentanoic acid } & MPFPeA \\
\hline & {$\left[{ }^{13} \mathrm{C}_{2}\right]$-Perfluorohexanoic acid } & MPFHxA \\
\hline & {$\left[{ }^{13} \mathrm{C}_{4}\right]$-Perfluoroheptanoic acid } & MPFHpA \\
\hline & {$\left[{ }^{13} \mathrm{C}_{4}\right]$-Perfluorooctanoic acid } & MPFOA \\
\hline & {$\left[{ }^{13} \mathrm{C}_{5}\right]$-Perfluorononanoic acid } & MPFNA \\
\hline & {$\left[{ }^{13} \mathrm{C}_{2}\right]$-Perfluorodecanoic acid } & MPFDA \\
\hline & {$\left[{ }^{13} \mathrm{C}_{2}\right]$-Perfluoroundecanoic acid } & MPFUnDA \\
\hline & {$\left[{ }^{13} \mathrm{C}_{2}\right]$-Perfluorododecanoic acid } & MPFDoDA \\
\hline & {$\left[{ }^{13} \mathrm{C}_{3}\right]$-Perfluorobutanesulfonic acid } & MPFBS \\
\hline & {$\left[{ }^{18} \mathrm{O}_{2}\right]$-Perfluorohexanesulfonic acid } & MPFHxS \\
\hline & {$\left[{ }^{13} \mathrm{C}_{4}\right]$-Perfluorooctanesulfonic acid } & MPFOS \\
\hline
\end{tabular}


Table S2. Optimized MS parameters for analysis of PFASs.

\begin{tabular}{|c|c|c|c|}
\hline Compounds & Parent ion $(\mathrm{m} / \mathrm{z})$ & Daughter ion $(\mathrm{m} / \mathrm{z})^{\mathrm{a}}$ & Collision energy $(\mathrm{V})$ \\
\hline \multicolumn{4}{|c|}{ Native PFASs } \\
\hline PFBA & 213 & 169 & 8 \\
\hline PFPeA & 263 & 219 & 5 \\
\hline PFHxA & 313 & $119, \mathbf{2 6 9}$ & 15,5 \\
\hline PFHpA & 363 & $169, \mathbf{3 1 9}$ & 16,9 \\
\hline PFOA & 413 & $169,219,369$ & $19,17,8$ \\
\hline PFNA & 463 & 219,419 & 18,10 \\
\hline PFDA & 513 & 269,469 & 17,8 \\
\hline PFUnA & 563 & 269,519 & 18,8 \\
\hline PFDoA & 613 & 269,569 & 20,10 \\
\hline PFTriDA & 663 & 169,619 & 35,11 \\
\hline PFBS & 299 & 80, 99 & 33,31 \\
\hline PFHxS & 399 & 80,99 & 38,35 \\
\hline PFOS & 499 & $\mathbf{8 0}, 99$ & 48,43 \\
\hline \multicolumn{4}{|c|}{ Internal standards } \\
\hline MPFBA & 217 & 172 & 8 \\
\hline MPFPeA & 266 & 222 & 5 \\
\hline MPFHxA & 315 & $119, \mathbf{2 7 0}$ & 15,10 \\
\hline MPFHpA & 367 & $169, \mathbf{3 2 2}$ & 25,24 \\
\hline MPFOA & 417 & 169,372 & 15,7 \\
\hline MPFNA & 468 & $219, \mathbf{4 2 3}$ & 16,10 \\
\hline MPFDA & 515 & 269,470 & 17,15 \\
\hline MPFUnA & 565 & $269, \mathbf{5 2 0}$ & 18,10 \\
\hline MPFDoA & 615 & $269, \mathbf{5 7 0}$ & 19,10 \\
\hline MPFBS & 302 & 80, 99 & 31,31 \\
\hline MPFHxS & 403 & $80,99, \mathbf{1 0 3}$ & $39,36,36$ \\
\hline MPFOS & 503 & 80, 99 & 49,41 \\
\hline
\end{tabular}

${ }^{\mathrm{a}}$ Daughter ions in bold font were used for quantification. 
Table S3. Method detection limits (MDLs) of PFASs in different sample types (ng/g $d w)$.

\begin{tabular}{lllll}
\hline Compounds & Soil $^{\mathrm{a}}$ & Plant $^{\mathrm{a}}$ & Muscle $^{\mathrm{a}}$ & Muscle $^{\mathrm{b}}$ \\
\hline PFBA & 0.059 & 0.031 & 0.060 & 0.375 \\
PFPeA & 0.048 & 0.040 & 0.049 & 0.250 \\
PFHxA & 0.059 & 0.053 & 0.041 & 0.150 \\
PFHpA & 0.062 & 0.021 & 0.045 & 0.500 \\
PFOA & 0.048 & 0.013 & 0.049 & 0.100 \\
PFNA & 0.010 & 0.045 & 0.034 & 0.100 \\
PFDA & 0.034 & 0.042 & 0.055 & 0.250 \\
PFUnDA & 0.093 & 0.036 & 0.042 & 0.100 \\
PFDoDA & 0.103 & 0.022 & 0.035 & 0.250 \\
PFTriDA & 0.078 & 0.057 & 0.061 & 0.150 \\
PFBS & 0.074 & 0.017 & 0.045 & 0.150 \\
PFHxS & 0.020 & 0.039 & 0.039 & 0.100 \\
PFOS & 0.107 & 0.104 & 0.038 & 0.100 \\
\hline
\end{tabular}

${ }^{\text {a }}$ MDL by triple quadruple MS.

${ }^{\mathrm{b}}$ MDL by Orbitrap MS. 
Table S4. Detection frequencies (\%) of PFASs in different sample types.

\begin{tabular}{llllll}
\hline Compounds & Soil & Plant & Pika muscle & Eagle muscle & Eagle feather \\
\hline PFBA & $55.6(5 / 9)$ & $54.5(6 / 11)$ & $57.1(8 / 14)$ & $95.5(21 / 22)$ & $86.4(19 / 22)$ \\
PFPeA & 0 & 0 & 0 & $9.1(2 / 22)$ & $68.2(15 / 22)$ \\
PFHxA & 0 & $18.2(2 / 11)$ & 0 & $13.6(3 / 22)$ & $59.1(13 / 22)$ \\
PFHpA & 0 & $9.1(1 / 11)$ & 0 & $36.4(8 / 22)$ & $50.0(11 / 22)$ \\
PFOA & 0 & 0 & $35.7(5 / 14)$ & $27.3(6 / 22)$ & $59.1(13 / 22)$ \\
PFNA & 0 & 0 & $42.9(6 / 14)$ & $40.9(9 / 22)$ & $77.3(17 / 22)$ \\
PFDA & 0 & $45.5(5 / 11)$ & $50.0(7 / 14)$ & $72.7(16 / 22)$ & $77.3(17 / 22)$ \\
PFUnDA & 0 & $45.5(5 / 11)$ & $42.9(6 / 14)$ & $63.6(14 / 22)$ & $81.8(18 / 22)$ \\
PFDoDA & 0 & $63.6(7 / 11)$ & $50.0(7 / 14)$ & $45.5(10 / 22)$ & $63.6(14 / 22)$ \\
PFTriDA & 0 & 0 & 0 & $36.4(8 / 22)$ & $72.7(16 / 22)$ \\
PFBS & $33.3(3 / 9)$ & $45.5(5 / 11)$ & $100.0(14 / 14)$ & $54.5(12 / 22)$ & $86.4(19 / 22)$ \\
PFHxS & $66.7(6 / 9)$ & $100.0(11 / 11)$ & $71.4(10 / 14)$ & $86.4(19 / 22)$ & $81.8(18 / 22)$ \\
PFOS & $22.2(2 / 9)$ & $100.0(11 / 11)$ & $71.4(10 / 14)$ & $100.0(22 / 22)$ & $77.3(17 / 22)$ \\
\hline
\end{tabular}


Table S5. Mean concentrations and concentration ranges of PFASs in different sample types (ng/g dw)

\begin{tabular}{|c|c|c|c|c|c|}
\hline Compounds & Soil & Plant & Pika muscle & Eagle muscle & Eagle feather \\
\hline PFBA & $0.060(<\mathrm{MDL}-0.094)$ & $0.102(<\mathrm{MDL}-0.188)$ & $1.345(<\mathrm{MDL}-5.761)$ & $2.626(<\mathrm{MDL}-6.706)$ & $1.806(<\mathrm{MDL}-5.352)$ \\
\hline PFPeA & $<\mathrm{MDL}$ & $<\mathrm{MDL}$ & $<\mathrm{MDL}$ & $(<\mathrm{MDL}-0.743)$ & $0.417(<\mathrm{MDL}-3.278)$ \\
\hline PFHxA & $<\mathrm{MDL}$ & $(<\mathrm{MDL}-0.159)$ & $<\mathrm{MDL}$ & $(<\mathrm{MDL}-1.042)$ & $0.399(<\mathrm{MDL}-1.229)$ \\
\hline PFHpA & $<\mathrm{MDL}$ & $(<\mathrm{MDL}-0.023)$ & $<\mathrm{MDL}$ & $(<\mathrm{MDL}-3.606)$ & $0.242(<\mathrm{MDL}-1.649)$ \\
\hline PFOA & $<\mathrm{MDL}$ & $<\mathrm{MDL}$ & $(<\mathrm{MDL}-1.052)$ & $(<\mathrm{MDL}-0.148)$ & $0.193(<\mathrm{MDL}-0.884)$ \\
\hline PFNA & $<\mathrm{MDL}$ & $<\mathrm{MDL}$ & $(<\mathrm{MDL}-0.070)$ & $(<\mathrm{MDL}-0.744)$ & $0.211(<\mathrm{MDL}-0.431)$ \\
\hline PFDA & $<\mathrm{MDL}$ & $(<\mathrm{MDL}-0.053)$ & $0.149(<\mathrm{MDL}-0.255)$ & $0.875(<\mathrm{MDL}-2.548)$ & $0.676(<\mathrm{MDL}-3.068)$ \\
\hline PFUnDA & $<\mathrm{MDL}$ & $(<\mathrm{MDL}-0.107)$ & $(<\mathrm{MDL}-0.278)$ & $0.761(<\mathrm{MDL}-1.677)$ & $0.660(<\mathrm{MDL}-2.518)$ \\
\hline PFDoDA & $<\mathrm{MDL}$ & $0.042(<\mathrm{MDL}-0.126)$ & $0.102(<\mathrm{MDL}-0.256)$ & $(<\mathrm{MDL}-0.357)$ & $0.383(<\mathrm{MDL}-1.779)$ \\
\hline PFTriDA & $<\mathrm{MDL}$ & $<\mathrm{MDL}$ & $<\mathrm{MDL}$ & $(<\mathrm{MDL}-0.176)$ & $0.202(<\mathrm{MDL}-4.494)$ \\
\hline PFBS & $(<\mathrm{MDL}-0.188)$ & $(<\mathrm{MDL}-0.022)$ & $0.559(0.223-1.047)$ & $1.141(<\mathrm{MDL}-3.439)$ & $0.809(<\mathrm{MDL}-3.046)$ \\
\hline PFHxS & $0.110(<\mathrm{MDL}-0.240)$ & $0.083(0.043-0.188)$ & $0.227(<\mathrm{MDL}-0.405)$ & $0.551(<\mathrm{MDL}-1.953)$ & $0.763(<\mathrm{MDL}-1.520)$ \\
\hline PFOS & $(<\mathrm{MDL}-1.054)$ & $0.383(0.117-0.832)$ & $0.866(<\mathrm{MDL}-1.615)$ & $15.205(1.145-69.003)$ & $5.848(<\mathrm{MDL}-29.637)$ \\
\hline$\sum$ PFASs & $0.427(0.130-1.507)$ & $0.634(0.406-1.085)$ & $2.451(0.601-8.293)$ & $22.003(5.021-76.482)$ & $12.729(3.160-43.700)$ \\
\hline
\end{tabular}

${ }^{a}$ For compound with detection frequencies less than $50 \%$, only concentration ranges were given. 
Table S6. Comparison of concentration results (ng/g dw) obtained from triple quadruple MS and Orbitrap MS in muscle samples.

\begin{tabular}{|c|c|c|c|c|c|c|c|c|}
\hline \multirow{3}{*}{ Compounds } & \multicolumn{2}{|l|}{ Pika muscle 1} & \multicolumn{2}{|l|}{ Pika muscle 2} & \multicolumn{2}{|l|}{ Eagle muscle 1} & \multicolumn{2}{|l|}{ Eagle muscle 2} \\
\hline & Triple quadruple & Orbitrap & Triple quadruple & Orbitrap & Triple quadruple & Orbitrap & Triple & Orbitrap \\
\hline & MS & MS & MS & MS & $\mathrm{MS}$ & MS & quadruple MS & MS \\
\hline PFBA & 5.761 & 6.031 & $<\mathrm{MDL}$ & $<\mathrm{MDL}$ & 1.513 & 1.622 & 6.706 & 6.955 \\
\hline PFPeA & $<\mathrm{MDL}$ & $<\mathrm{MDL}$ & $<\mathrm{MDL}$ & $<\mathrm{MDL}$ & $<\mathrm{MDL}$ & $<\mathrm{MDL}$ & 0.743 & 0.801 \\
\hline PFHxA & $<\mathrm{MDL}$ & $<\mathrm{MDL}$ & $<\mathrm{MDL}$ & $<\mathrm{MDL}$ & $<\mathrm{MDL}$ & $<\mathrm{MDL}$ & $<\mathrm{MDL}$ & $<\mathrm{MDL}$ \\
\hline PFHpA & $<\mathrm{MDL}$ & $<\mathrm{MDL}$ & $<\mathrm{MDL}$ & $<\mathrm{MDL}$ & $<\mathrm{MDL}$ & $<\mathrm{MDL}$ & $<\mathrm{MDL}$ & $<\mathrm{MDL}$ \\
\hline PFOA & 0.056 & $<\mathrm{MDL}$ & 0.067 & $<\mathrm{MDL}$ & 0.141 & 0.150 & 0.091 & $<\mathrm{MDL}$ \\
\hline PFNA & 0.070 & $<\mathrm{MDL}$ & 0.102 & 0.108 & 0.744 & 0.693 & $<\mathrm{MDL}$ & $<\mathrm{MDL}$ \\
\hline PFDA & $<\mathrm{MDL}$ & $<\mathrm{MDL}$ & 0.171 & 0.164 & 2.548 & 2.796 & 0.604 & 0.560 \\
\hline PFUnDA & $<\mathrm{MDL}$ & $<\mathrm{MDL}$ & 0.144 & 0.133 & 1.407 & 1.396 & 0.604 & 0.650 \\
\hline PFDoDA & 0.106 & $<\mathrm{MDL}$ & 0.177 & $<\mathrm{MDL}$ & 0.357 & 0.347 & $<\mathrm{MDL}$ & $<\mathrm{MDL}$ \\
\hline PFTriDA & $<\mathrm{MDL}$ & $<\mathrm{MDL}$ & $<\mathrm{MDL}$ & $<\mathrm{MDL}$ & 0.124 & $<\mathrm{MDL}$ & 0.101 & $<\mathrm{MDL}$ \\
\hline $\mathrm{PFBS}^{\mathrm{a}}$ & $0.450(0.446)$ & 0.447 & $0.489(0.453)$ & 0.452 & $0.046(<\mathrm{MDL})$ & $<\mathrm{MDL}$ & $0.957(0.964)$ & 1.051 \\
\hline $\mathrm{PFHxS}^{\mathrm{a}}$ & $0.155(0.153)$ & 0.167 & $0.155(0.162)$ & 0.158 & $0.177(0.168)$ & 0.172 & $0.163(0.159)$ & 0.171 \\
\hline $\mathrm{PFOS}^{\mathrm{a}}$ & $<\operatorname{MDL}(<\mathrm{MDL})$ & $<\mathrm{MDL}$ & $1.228(1.216)$ & 1.316 & $69.003(71.317)$ & 64.953 & $9.625(9.857)$ & 10.100 \\
\hline
\end{tabular}

${ }^{a}$ Concentrations in brackets were quantified using $99 \mathrm{~m} / \mathrm{z}$ as quantification ion. 
Table S7. Stable isotopes and trophic levels of the plant-pika-eagle food chain.

\begin{tabular}{lllll}
\hline Species & $\mathrm{N}$ & $\delta^{13} \mathrm{C}(\%)$ & $\delta^{15} \mathrm{~N}(\%)$ & $\mathrm{TL}$ \\
\hline Plant & 11 & $-25.97 \pm 0.59$ & $-1.58 \pm 0.77$ & $1.00 \pm 0.18$ \\
Pika & 14 & $-25.91 \pm 1.56$ & $2.43 \pm 0.51$ & $2.07 \pm 0.12$ \\
Eagle & 11 & $-25.07 \pm 1.07$ & $5.19 \pm 0.50$ & $2.84 \pm 0.13$ \\
\hline
\end{tabular}


Table S8. TMFs of PFASs in different types of food webs in different regions.

\begin{tabular}{|c|c|c|c|c|c|c|c|c|c|c|c|c|c|c|c|}
\hline $\begin{array}{l}\text { Food web } \\
\text { type }\end{array}$ & $\begin{array}{l}\text { Top } \\
\text { predator }\end{array}$ & PFBA & PFPeA & $\begin{array}{l}\text { PFHx } \\
\text { A }\end{array}$ & $\begin{array}{l}\text { PFHp } \\
\text { A }\end{array}$ & PFOA & PFNA & PFDA & $\begin{array}{l}\text { PFUn } \\
\text { DA }\end{array}$ & $\begin{array}{l}\text { PFDo } \\
\text { DA }\end{array}$ & $\begin{array}{l}\text { PFTri } \\
\text { DA }\end{array}$ & PFBS & PFHxS & PFOS & $\begin{array}{l}\text { Refer } \\
\text { ence }\end{array}$ \\
\hline Marine & Whale & $\mathrm{-a}^{\mathrm{a}}$ & - & - & 1.43 & 3.28 & 7.03 & 8.29 & 7.98 & 4.79 & - & - & - & 17.4 & 7 \\
\hline Marine & Fish & $\mathrm{NA}^{\mathrm{b}}$ & NA & - & NA & - & $\begin{array}{l}1.85- \\
2.11\end{array}$ & $\begin{array}{l}2.21- \\
2.89\end{array}$ & $\begin{array}{l}1.46- \\
1.52\end{array}$ & $\begin{array}{l}2.28- \\
4.07\end{array}$ & $\begin{array}{l}3.54- \\
4.40\end{array}$ & - & - & $\begin{array}{l}3.83- \\
3.88\end{array}$ & 8 \\
\hline Marine & Fish & - & - & - & - & 0.35 & 0.28 & 0.92 & 0.72 & 0.58 & 1.06 & - & - & 1.76 & 9 \\
\hline Marine & Fish & 0.49 & - & 0.02 & - & 0.2 & - & - & 0.76 & - & 0.75 & 0.4 & - & 1.57 & 10 \\
\hline Estuary & Fish & - & - & - & - & 6.0 & 3.1 & 1.7 & 1.8 & 1.1 & 0.66 & - & 4.3 & 3.9 & 11 \\
\hline Estuary & Fish & - & - & - & - & 1.0 & 0.88 & 0.96 & 0.93 & 1.3 & 0.96 & - & 1.5 & 1.5 & 11 \\
\hline Estuary & Fish & NA & - & - & - & - & 1.34 & - & 0.41 & 0.94 & 0.39 & - & - & 1.53 & 12 \\
\hline Lake & Fish & NA & NA & - & - & 2.13 & 2.19 & 2.53 & 2.25 & 3.19 & NA & - & - & 3.74 & 13 \\
\hline River & Fish & - & - & - & 0.75 & 0.81 & - & 0.83 & - & - & - & 1.08 & - & 0.93 & 14 \\
\hline Terrestrial & Wolf & NA & NA & - & - & $1.1-1.3$ & $1.9-2.7$ & $2.3-2.6$ & $2.2-2.9$ & $1.3-2.2$ & $1.4-2.0$ & NA & - & $2.2-2.6$ & 15 \\
\hline Terrestrial & Eagle & 5.11 & - & - & - & - & - & 4.65 & 4.37 & 1.84 & - & 5.96 & 2.43 & 5.75 & $\begin{array}{l}\text { This } \\
\text { study }\end{array}$ \\
\hline
\end{tabular}

${ }^{a}$ PFASs analyzed but not detected $(<\mathrm{MDL})$ in organisms.

${ }^{\mathrm{b}}$ PFASs not analyzed in organisms. 

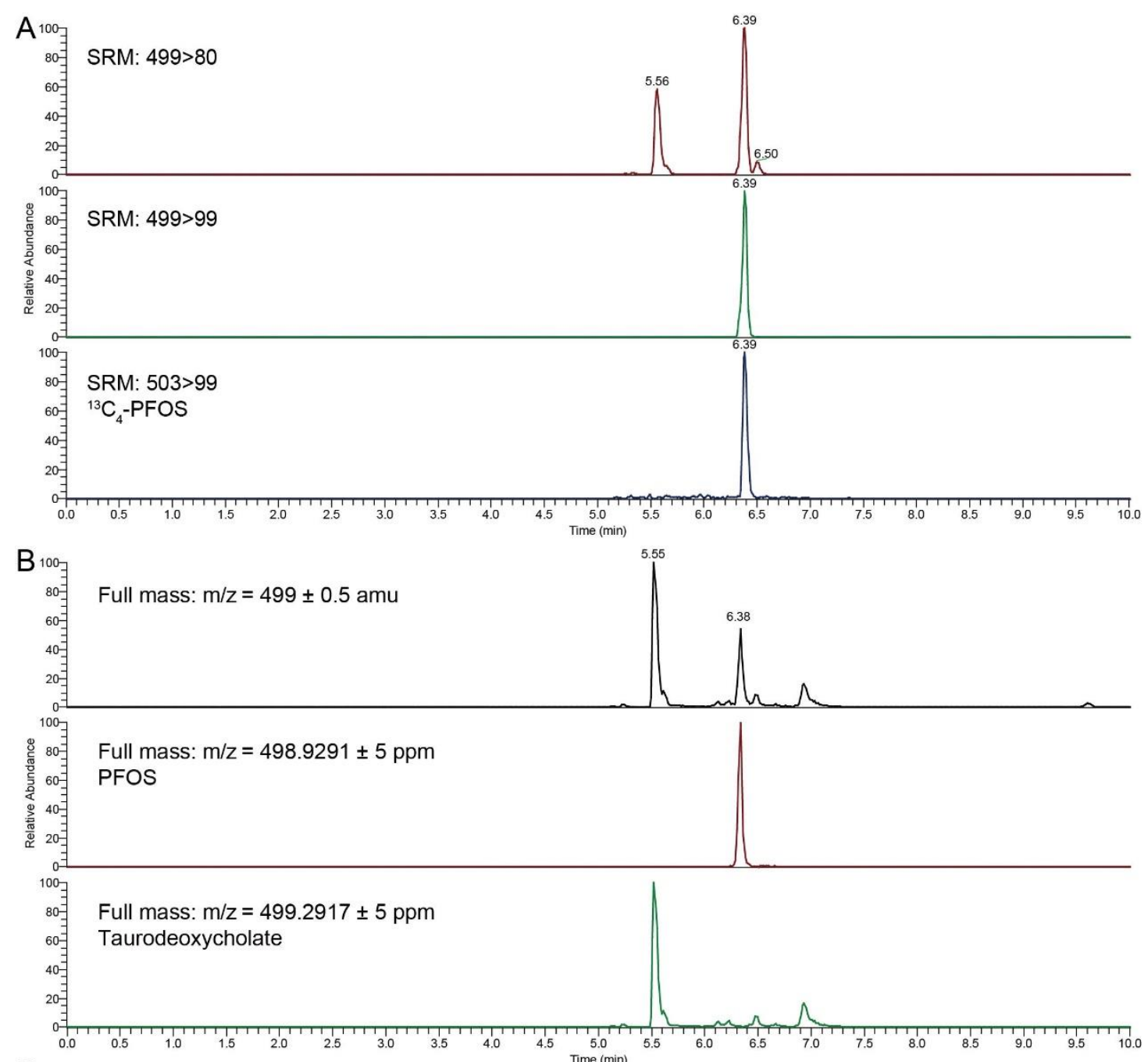

C

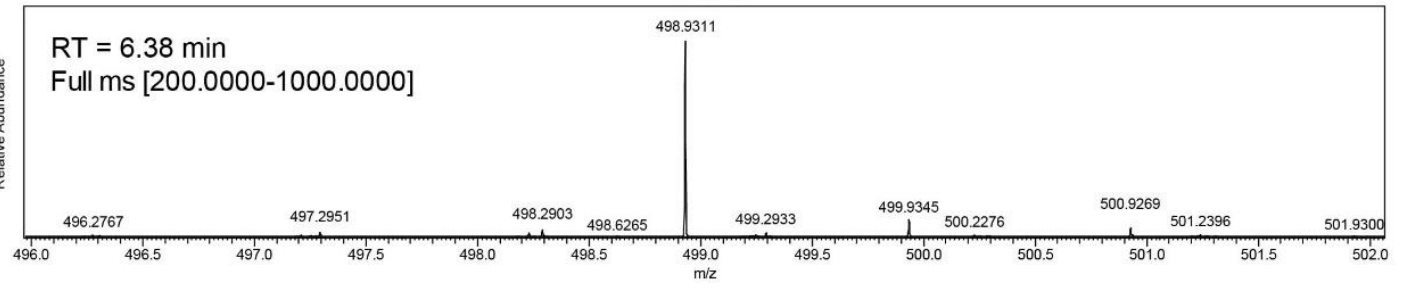

Figure S1. Confirmation analysis of PFOS in muscle samples by Orbitrap MS. (A) Extracted ion chromatograms of PFOS and ${ }^{13} \mathrm{C}_{4}$-PFOS on triple quadruple MS. (B) Extracted ion chromatograms of PFOS and interfering ions on Orbitrap MS. (C) Full mass scan of the peak at 6.38 min on Orbitrap MS. 

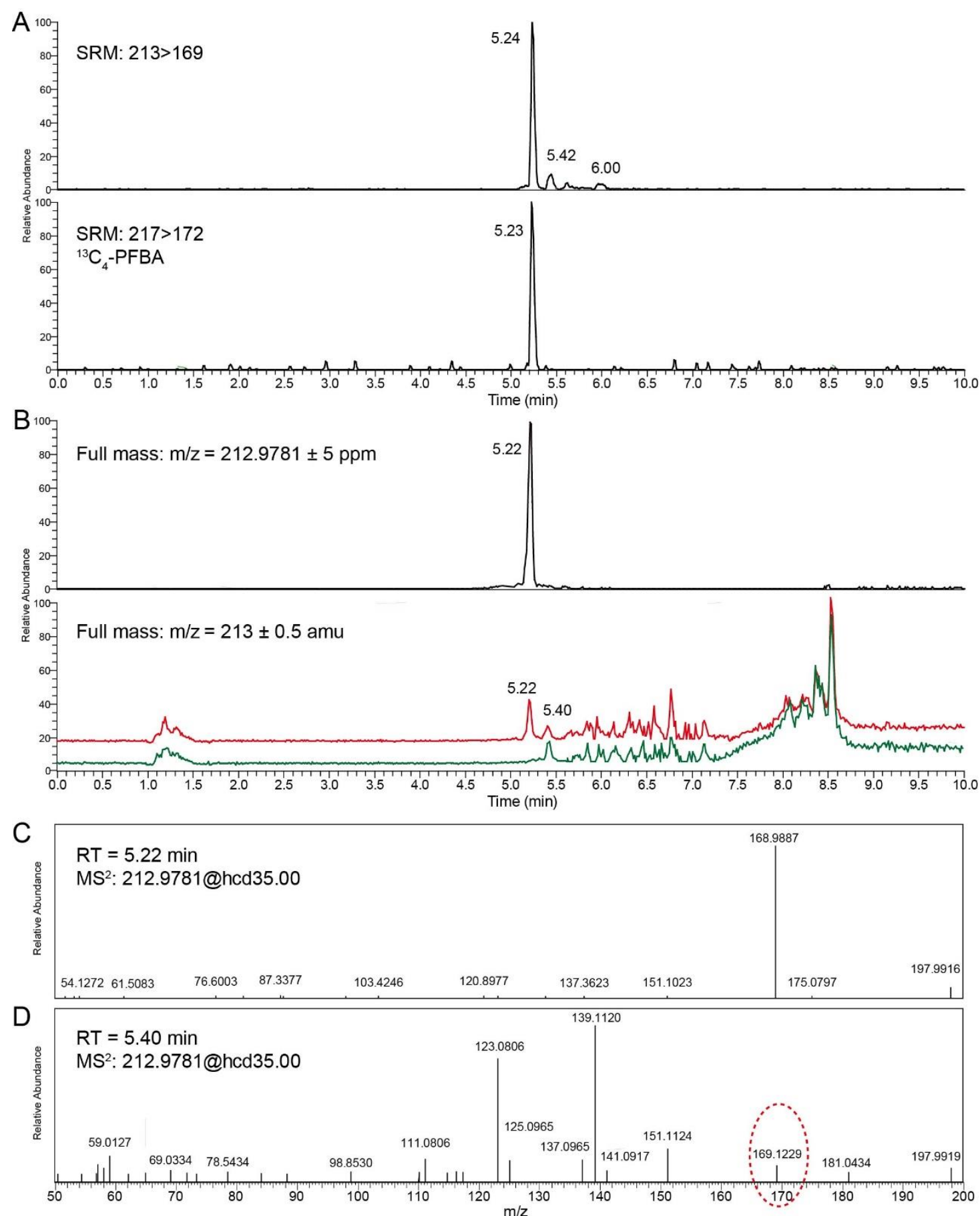

Figure S2. Confirmation analysis of PFBA in muscle samples by Orbitrap MS. (A) Extracted ion chromatograms of PFBA and ${ }^{13} \mathrm{C}_{4}$-PFBA on triple quadruple MS. (B) Extracted ion chromatograms of PFBA and interfering ions on Orbitrap MS. Red: sample with PFBA; green: sample without PFBA. (C) $\mathrm{MS}^{2}$ scan of the peak at $5.22 \mathrm{~min}$ on Orbitrap MS. (D) $\mathrm{MS}^{2}$ scan of the peak at 5.40 min on Orbitrap MS. 

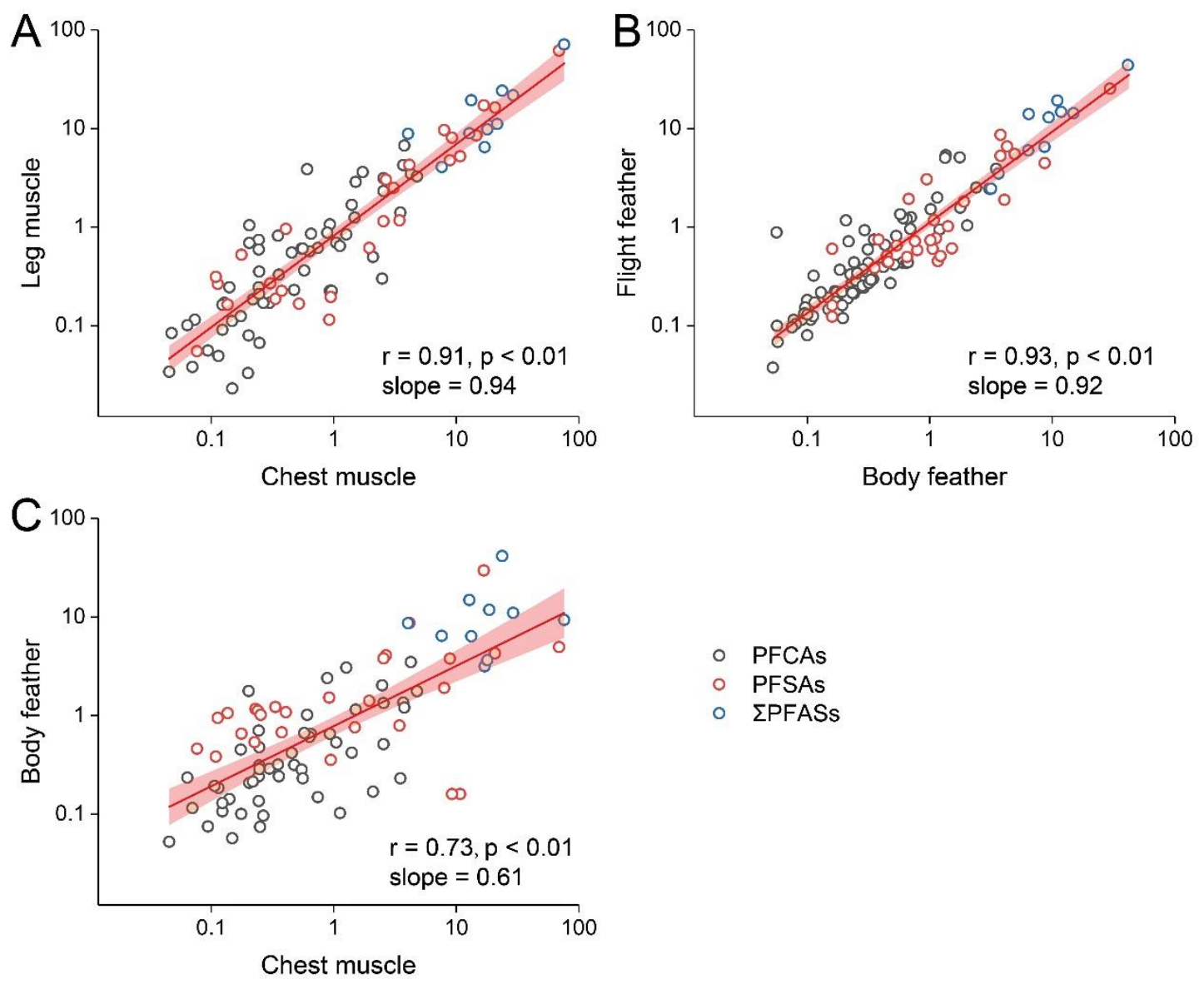

Figure S3. Correlations between PFASs concentrations (ng/g dw) in eagle muscles and feathers. (A) Correlations of PFASs between chest and leg muscles. (B) Correlations of PFASs between body and flight feathers. (C) Correlations of PFASs between chest muscles and body feathers. 


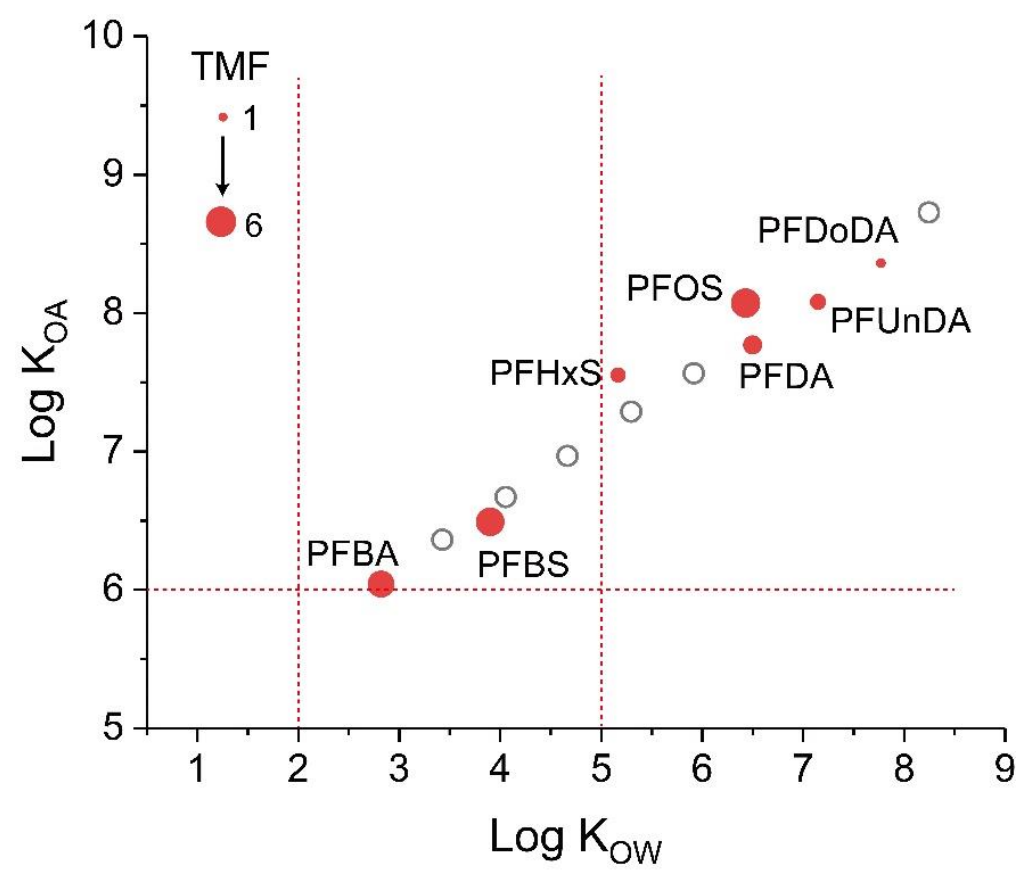

Figure S4. Relationships between TMFs and physicochemical properties. The size of red dots represents the value of TMFs. TMFs are missing for grey circles. Values of Log Kow and Log KOA of PFASs were obtained from the literature. ${ }^{16}$ Chemicals with either of the following characteristics suggest biomagnification potential in terrestrial food webs: (1) $\log \mathrm{K}_{\mathrm{OW}} \geq 5$; (2) $2<\log \mathrm{K}_{\mathrm{OW}}<5$ and $\log \mathrm{K}_{\mathrm{OA}} \geq 6$. $^{17}$ 


\section{References}

(1) Li, Y.; Gao, K.; Duo, B.; Zhang, G.; Cong, Z.; Gao, Y.; Fu, J.; Zhang, A.; Jiang, G. Analysis of a broad range of perfluoroalkyl acids in accipiter feathers: method optimization and their occurrence in Nam Co Basin, Tibetan Plateau. Environ. Geochem. Health 2018, 40, (5), 1877-1886.

(2) Benskin, J. P.; Bataineh, M.; Martin, J. W. Simultaneous Characterization of Perfluoroalkyl Carboxylate, Sulfonate, and Sulfonamide Isomers by Liquid Chromatography-Tandem Mass Spectrometry. Anal. Chem. 2007, 79, (17), 6455-6464.

(3) Bangma, J. T.; Reiner, J.; Fry, R. C.; Manuck, T.; McCord, J.; Strynar, M. J. Identification of an Analytical Method Interference for Perfluorobutanoic Acid in Biological Samples. Environ. Sci. Technol. Lett. 2021, 8, (12), 1085-1090.

(4) Jardine, T. D.; Kidd, K. A.; Fisk, A. T. Applications, Considerations, and Sources of Uncertainty When Using Stable Isotope Analysis in Ecotoxicology. Environ. Sci. Technol. 2006, 40, (24), 7501-7511.

(5) Fisk, A. T.; Hobson, K. A.; Norstrom, R. J. Influence of Chemical and Biological Factors on Trophic Transfer of Persistent Organic Pollutants in the Northwater Polynya Marine Food Web. Environ. Sci. Technol. 2001, 35, (4), 732-738.

(6) Morris, A. D.; Muir, D. C. G.; Solomon, K. R.; Teixeira, C.; Duric, M.; Wang, X. Trophodynamics of current use pesticides and ecological relationships in the Bathurst region vegetation-caribou-wolf food chain of the Canadian Arctic. Environ. Toxicol. Chem. 2014, 33, (9), 1956-1966.

(7) Kelly, B. C.; Ikonomou, M. G.; Blair, J. D.; Surridge, B.; Hoover, D.; Grace, R.; Gobas, F. A. P. C. Perfluoroalkyl Contaminants in an Arctic Marine Food Web: Trophic Magnification and Wildlife Exposure. Environ. Sci. Technol. 2009, 43, (11), 4037-4043. (8) Liu, Y.; Ruan, T.; Lin, Y.; Liu, A.; Yu, M.; Liu, R.; Meng, M.; Wang, Y.; Liu, J.; Jiang, G. Chlorinated Polyfluoroalkyl Ether Sulfonic Acids in Marine Organisms from Bohai Sea, China: Occurrence, Temporal Variations, and Trophic Transfer Behavior. Environ. Sci. Technol. 2017, 51, (8), 4407-4414.

(9) Pan, C. G.; Xiao, S. K.; Yu, K. F.; Wu, Q.; Wang, Y. H. Legacy and alternative perand polyfluoroalkyl substances in a subtropical marine food web from the Beibu Gulf, 
South China: Fate, trophic transfer and health risk assessment. J. Hazard. Mater. 2021, $403,123618$.

(10) Du, D.; Lu, Y.; Zhou, Y.; Li, Q.; Zhang, M.; Han, G.; Cui, H.; Jeppesen, E. Bioaccumulation, trophic transfer and biomagnification of perfluoroalkyl acids (PFAAs) in the marine food web of the South China Sea. J. Hazard. Mater. 2021, 405, 124681. (11) Munoz, G.; Budzinski, H.; Babut, M.; Drouineau, H.; Lauzent, M.; Menach, K. L.; Lobry, J.; Selleslagh, J.; Simonnet-Laprade, C.; Labadie, P. Evidence for the Trophic Transfer of Perfluoroalkylated Substances in a Temperate Macrotidal Estuary. Environ. Sci. Technol. 2017, 51, (15), 8450-8459.

(12) Miranda, D. A.; Benskin, J. P.; Awad, R.; Lepoint, G.; Leonel, J.; Hatje, V. Bioaccumulation of Per- and polyfluoroalkyl substances (PFASs) in a tropical estuarine food web. Sci. Total Environ. 2021, 754, 142146.

(13) Fang, S.; Chen, X.; Zhao, S.; Zhang, Y.; Jiang, W.; Yang, L.; Zhu, L. Trophic Magnification and Isomer Fractionation of Perfluoroalkyl Substances in the Food Web of Taihu Lake, China. Environ. Sci. Technol. 2014, 48, (4), 2173-2182.

(14) Penland, T. N.; Cope, W. G.; Kwak, T. J.; Strynar, M. J.; Grieshaber, C. A.; Heise, R. J.; Sessions, F. W. Trophodynamics of Per- and Polyfluoroalkyl Substances in the Food Web of a Large Atlantic Slope River. Environ. Sci. Technol. 2020, 54, (11), 68006811.

(15) Müller, C. E.; De Silva, A. O.; Small, J.; Williamson, M.; Wang, X.; Morris, A.; Katz, S.; Gamberg, M.; Muir, D. C. G. Biomagnification of Perfluorinated Compounds in a Remote Terrestrial Food Chain: Lichen-Caribou-Wolf. Environ. Sci. Technol. 2011, $45,(20), 8665-8673$.

(16) Wang, Z.; MacLeod, M.; Cousins, I. T.; Scheringer, M.; Hungerbühler, K. Using COSMOtherm to predict physicochemical properties of poly- and perfluorinated alkyl substances (PFASs). Environ. Chem. 2011, 8, (4), 389-398.

(17) Kelly, B. C.; Ikonomou, M. G.; Blair, J. D.; Morin, A. E.; Gobas, F. A. P. C. Food Web-Specific Biomagnification of Persistent Organic Pollutants. Science 2007, 317, (5835), 236-239. 